廿]

'Dept of Thoracic Medicine, Heraklion University Hospital, Medical School, University of Crete,

Heraklion, Greece.

2Dept of Rheumatology, Heraklion University Hospital, Medical School, University of Crete,

Heraklion, Greece.

\title{
New respiratory symptoms and lung imaging findings in a woman with polymyositis
}

\section{Case report}

Cite as: Bolaki $M$,

Karagiannis K, Bertsias G, et al. New respiratory symptoms and lung imaging findings in a woman with polymyositis. Breathe 2018; 14: e34-e39.
A 57-year-old Greek woman presented with a 5-day history of fever and progressively worse shortness of breath without any additional symptoms.

Her past medical history included polymyositis since 2008 for which she had received various treatment regimens (e.g. azathioprine, cyclosporine) that had been stopped due to liver toxicity. Subsequently, she had been treated with methotrexate and corticosteroids for the past few years. 1 month ago, she was commenced on rituximab by her rheumatologist due to a high suspicion of respiratory muscles impairment (compatible pulmonary function tests and new onset hypercapnic respiratory failure) over the past few months. The patient's history was also remarkable for paroxysmal atrial fibrillation, depressive disorder, hypothyroidism and hypoparathyroidism for which she was on regular treatments. She was also an ex-smoker with a smoking history of 40 pack-years.

On clinical examination the patient was haemodynamically stable with a mildly elevated body temperature $\left(38^{\circ} \mathrm{C}\right)$. On auscultation there were non-musical sounds (crackles) at both lung bases, mostly on the right side, which had not been present on previous examinations. The rest of clinical examination was unremarkable.

Laboratory tests revealed elevated inflammatory markers (C-reactive protein $=11.5 \mathrm{mg} \cdot \mathrm{dL}^{-1}$, white blood cells $=7100$ cells $\mu \mathrm{L}^{-1}$, erythrocyte sedimentation rate $=31 \mathrm{~mm} \cdot \mathrm{h}^{-1}$ ) without any other remarkable abnormality. The arterial blood gases were indicative of hypercapnic respiratory failure: oxygen tension $=52 \mathrm{mmHg}$, carbon dioxide tension $=49 \mathrm{mmHg}, \mathrm{pH}=7.37, \mathrm{HCO}_{3}{ }^{-}=6 \mathrm{mEq} \cdot \mathrm{L}^{-1}$ on room air (inspiratory oxygen fraction=21\%). The chest radiograph didn't show any obvious abnormality (figure 1).

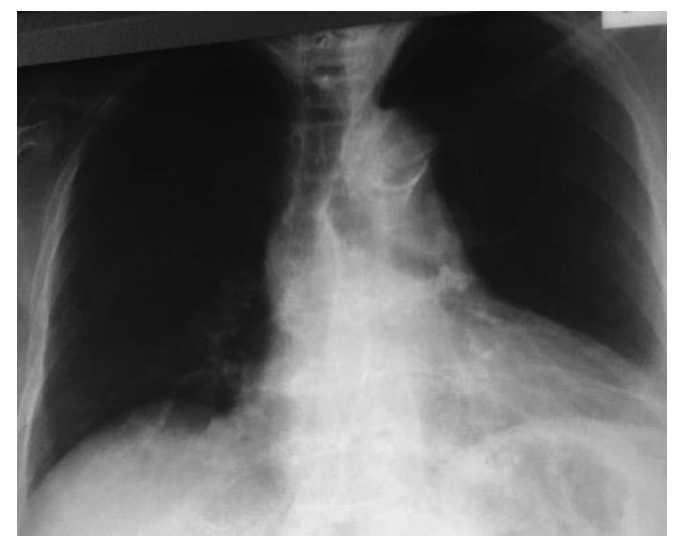

Figure 1 Chest radiograph of the patient at presentation. There is no obvious abnormality in the lung parenchyma. 


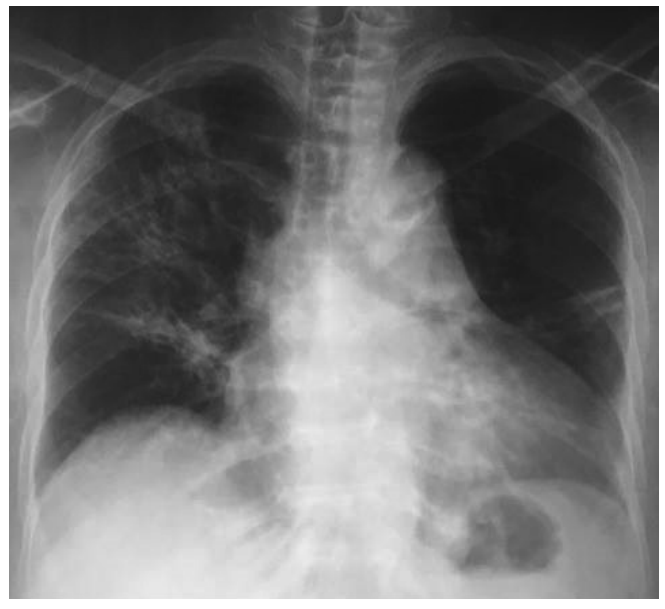

Figure 2 Chest radiograph of the patient some days after presentation. Lung opacities mainly located in the right upper lobe can be seen.

The patient was initially managed with empirical antibiotics (ceftriaxone and azithromycin) and oxygen therapy. However, she deteriorated with tachypnoea, an altered level of consciousness, and worsening respiratory failure with the need for noninvasive mechanical ventilation. A new chest radiograph showed findings on the right upper lobe not previously recognised (figure 2) and neutropenia was also revealed by blood tests.

\section{Task 1}

What further action would you take based on the clinical presentation?

\section{Answer 1}

Transfer to the intensive care unit (ICU) and endotracheal intubation would be the first step.

The patient was transferred to the ICU and prompt endotracheal intubation was considered due to the rapid deterioration of the clinical picture and gas exchange. Antibiotic treatment was escalated to meropenem, linezolid and trimethoprim/ sulfamethoxazole, and the patient was intubated the following day.

\section{Task 2}

What would be your next step for diagnosis and further management? 


\section{Answer 2}

High-resolution computed tomography (HRCT) scan of the chest in order to investigate the new findings on the chest radiograph and shed light on the rapid deterioration of the patient.

On admission to the ICU the patient underwent HRCT (figure 3), which showed bilateral interstitial thickening and ground-glass opacities. On the right upper lobe the changes were more prominent and
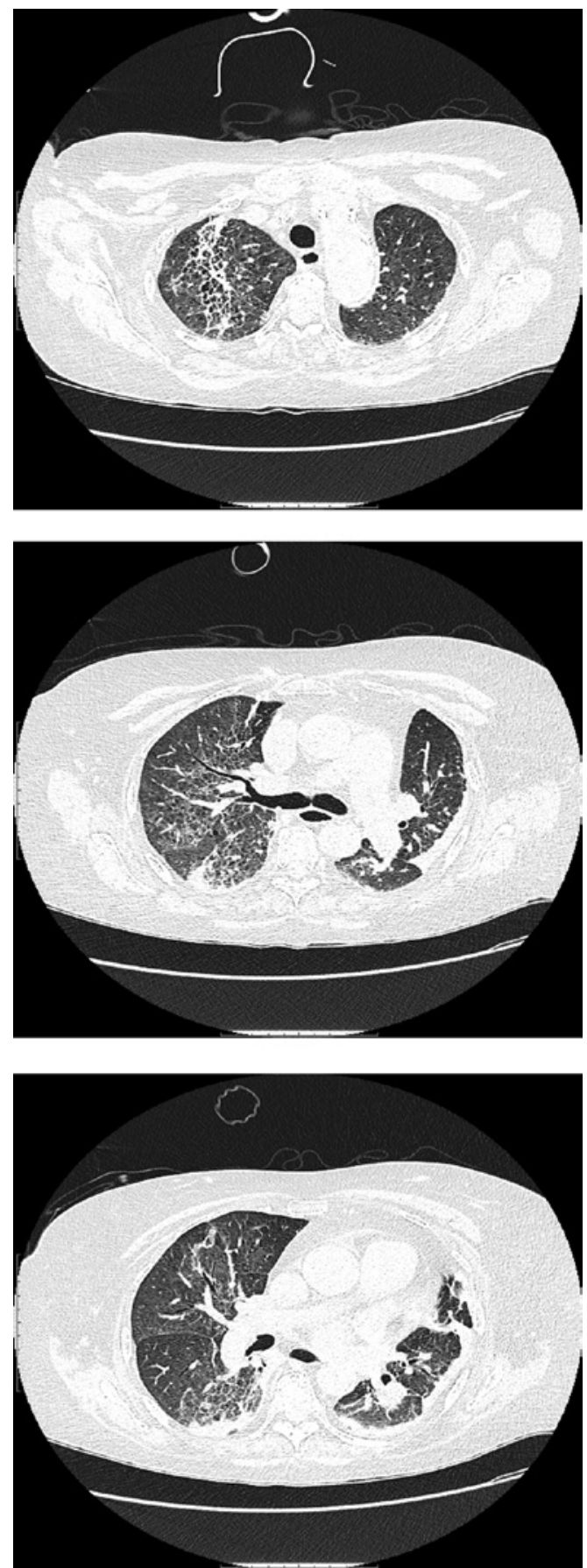

Figure 3 HRCT on admission to the ICU.
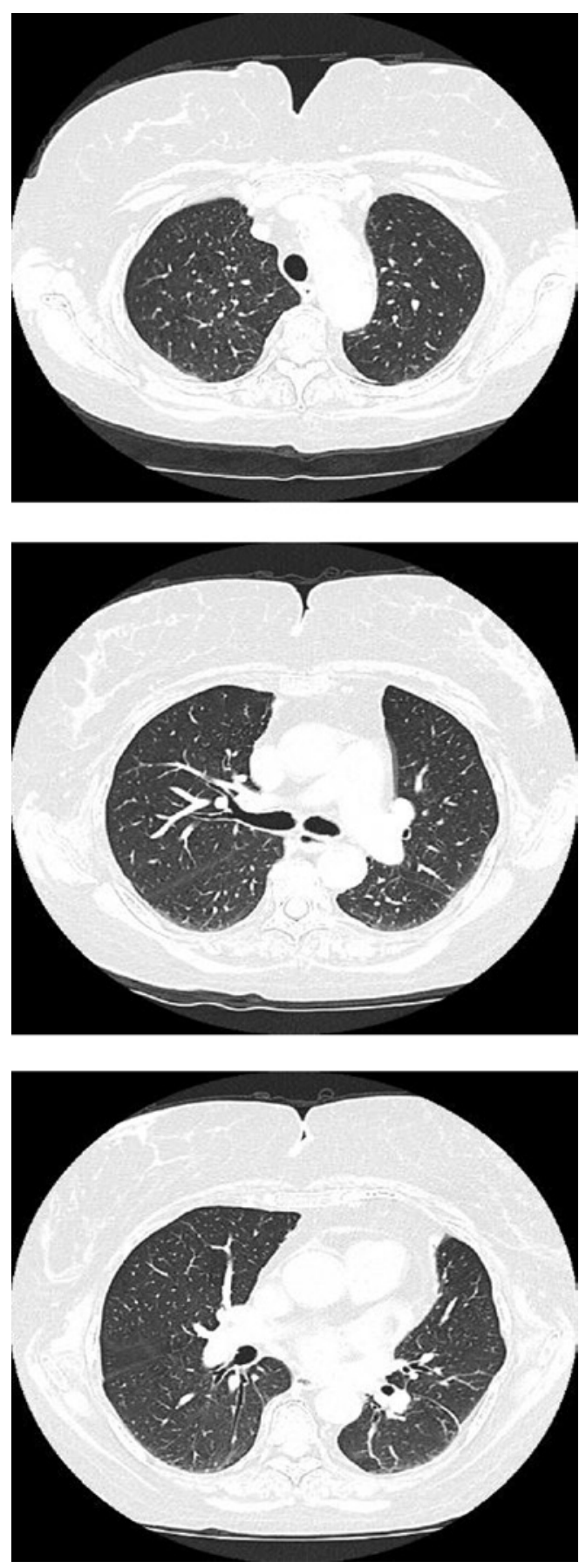

Figure 4 HRCT performed less than a month prior to presentation.

there were also bronchiectasis lesions. Such findings had not been present in a prior HRCT performed less than a month previously in the context of the investigation of the new onset of respiratory failure (figure 4).

\section{Task 3}

What could be the differential diagnoses based on the clinical presentation and imaging findings? 


\section{Answer 3}

1) Lower respiratory tract infection in an immunocompromised patient; 2) drug-induced interstitial lung disease (ILD) (rituximab toxicity); 3) connective tissue disease-related ILD associated with polymyositis; and 4) other diagnoses (organising pneumonia, eosinophilic pneumonia, diffuse alveolar haemorrhage, hypersensitivity pneumonitis)

The new-onset ground-glass opacities mainly located in the right upper lobe could be consistent with infection due to either nonspecific (e.g. bacteria or viruses) or specific pathogens (e.g. mycobacteria, fungus or Pneumonocystis jirovecii). Urine and blood cultures were negative and subsequently a bronchoscopy was performed. Cultures of bronchial secretions samples for bacteria, mycobacteria, fungi and Pneumocystis jirovecii pneumonia were also negative. Finally, cytology of bronchial washing samples did not reveal any malignant cells, whereas bronchoalveolar lavage (BAL) cell count showed a predominantly lymphocytic fluid (48\% lymphocytes, $32 \%$ macrophages, $18 \%$ neutrophils and 2\% eosinophils).

Drug-induced ILD, particularly rituximab toxicity, is rather probable based on the course of the symptoms, which are correlated with the administration of the drug. In addition, the radiological findings are compatible with lung toxicity due to anti-CD20 treatment, while BAL lymphocytosis is another hint pointing towards a diagnosis of rituximab-induced ILD.

Although polymyositis-related ILD cannot be entirely excluded, there are clues directing against this diagnosis. Specifically, the patient had been diagnosed with polymyositis since 2008 without pulmonary parenchymal involvement until her presentation. Furthermore, the radiological findings of the present case are not common in patients with polymyositis, as peribronchovascular and perilobular lower lobe predominant ground glass and consolidation changes represent the main HRCT findings in polymyositis-related ILD.

Other diagnoses (e.g. organising pneumonia, eosinophilic pneumonia, diffuse alveolar haemorrhage or hypersensitivity pneumonitis) come lower down the differential diagnosis list either due to the clinical or radiological features.

\section{Task 4}

What would be your working diagnosis based on the above results? 


\section{Answer 4}

Rituximab-induced ILD.

Microbiological analysis of BAL fluid can safely exclude the vast majority of respiratory tract infections. The obvious correlation of clinical features with the onset of treatment along with the radiological findings and BAL lymphocytosis justify rituximab-induced ILD as the working diagnosis in this case. Transbronchial biopsy was not performed as the risk/benefit ratio was not acceptable for the patient.

Antibiotics were discontinued and the patient was managed with corticosteroid pulses for 3 days, followed by gradually tapering oral corticosteroids. She was also started on a prophylactic dose of trimethoprim/sulfamethoxazole. The rituximab was permanently discontinued. The patient improved remarkably and was extubated 5 days later. A new HRCT scan 2 months later revealed almost complete resolution of the abnormal findings (figure 5 ).

\section{Discussion}

Idiopathic inflammatory myopathies constitute a rare group of connective tissue diseases in which lung involvement is a great challenge [1]. Rituximab is a monoclonal antibody directed against the CD-20 antigen on the surface of pre-B-cells and is increasingly used in cases of idiopathic inflammatory myopathies which are refractory to first-line therapies [2]. Currently, rituximab is the most promising biological therapy for refractory myositis. The rate of response to rituximab was estimated to be $78.3 \%$ and it has been described that patients with autoantibodies are more likely to respond to rituximab therapy [3].

The incidence of rituximab-induced ILD is approximately $0.01-0.03 \%$. The most common indication for rituximab is haematological malignancy and it is usually part of combination chemotherapy. Rituximab-induced ILD occurs more commonly in male patients, in fifth and sixth decade of life, during the fourth cycle of treatment and 30 days after the last dose. The clinical presentation is nonspecific and the usual imaging pattern consists of diffuse bilateral lung infiltrates. Hypoxaemia is very common and the pulmonary function tests usually reveal a restrictive pattern with reduced diffusion capacity. The BAL fluid shows most commonly lymphocytosis and the pathological findings on lung biopsy vary widely [4].

The causal relationship between rituximab and lung disease follows the criteria generally used to evaluate drug-induced lung disease. These include: 1) timing, 2) clinical manifestations, 3) exclusion of other causes, 4) favourable outcome following discontinuation of the suspected drug, and 5) positive results following accidental or intentional rechallenge [5]. The treatment of rituximab-induced ILD is based on the following principles: 1) discontinuation
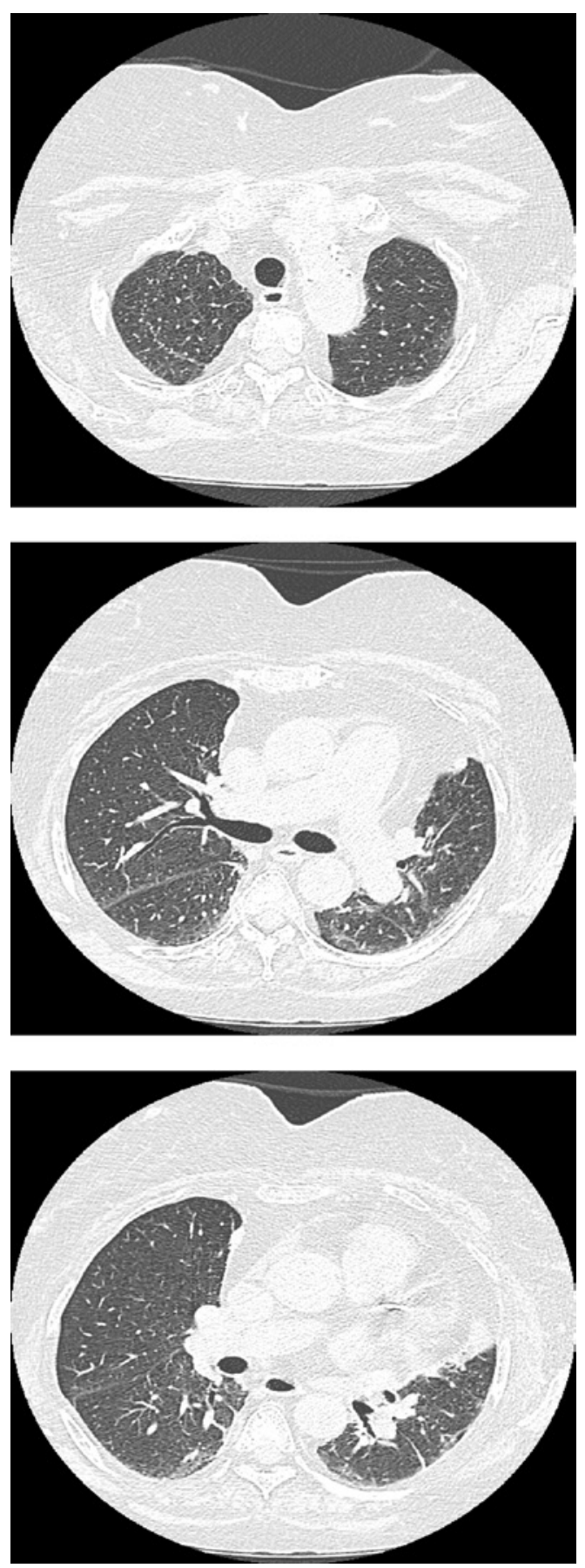

Figure 5 HRCT performed 2 months later revealing almost complete resolution of the abnormal findings.

of the offensive agent, 2) supportive treatment and 3) corticosteroid treatment, although a firm recommendation regarding the administration of steroids does not exist [6].

In conclusion, given the fact that rituximab is increasingly prescribed in various diseases, doctors should be aware of the ILD this agent can potentially induce. The suspicion should arise in patients receiving rituximab who appear with new respiratory symptoms or lung imaging findings. We present a rare case of rituximab-induced ILD in a patient with polymyositis. 


\section{Conflict of interest}

None declared.

\section{References}

1. Lega JC, Reynaud Q, Belot A, et al. Idiopathic inflammatory myopathies and the lung. Eur Respir Rev 2015 24: 216-238.

2. Mandel DE, Malemud CJ, Askari AD. Idiopathic inflammatory myopathies: a review of the classification and impact of pathogenesis. Int J Mol Sci 2017; 18: 1084.

3. Fasano S, Alves SC, Isenberg DA. Current pharmacological treatment of idiopathic inflammatory myopathies. Expert Rev Clin Pharmacol 2016; 9: 547-558.
4. Hadjinikolaou AV, Nisar VK, Parfrey $\mathrm{H}$, et al. Non-infectious pulmonary toxicity of rituximab: a systematic review. Rheumatology 2012; 51: 653-662.

5. Liote $\mathrm{H}$, Liote F, Seroussi B, et al. Rituximab-induced lung disease: a systematic literature review. Eur Respir J 2010; 35: 681-687.

6. Naqibullah M, Shaker SB, Bach KS, et al. Rituximab-induced interstital lung disease: five case reports. Eur Clin RespirJ 2015; 2: 27178. 